\title{
Association of MBOAT7-TMC4 rs641738 with the risk of hepatocellular carcinoma and persistent infection of hepatitis $B$ virus: a case-control study and meta-analysis
}

\section{Peng Wang}

Tongji Hospital of Tongji Medical College of Huazhong University of Science and Technology

Yu Zhou

Tongji Hospital of Tongji Medical College of Huazhong University of Science and Technology

Lu Li

Shantou University Medical College

\section{Yajie Gong}

Huazhong University of Science and Technology Tongji Medical College

\section{Rong Zhong}

Huazhong University of Science and Technology Tongji Medical College

Na Shen ( $\nabla$ shenna@tjh.tjmu.edu.cn )

Tongji Hospital of Tongji Medical College of Huazhong University of Science and Technology https://orcid.org/0000-0003-4362-9207

\section{Research article}

Keywords: Hepatocellular carcinoma (HCC), persistent HBV infection, rs641738, susceptibility

Posted Date: October 24th, 2019

DOI: https://doi.org/10.21203/rs.2.16438/v1

License: @ (1) This work is licensed under a Creative Commons Attribution 4.0 International License. Read Full License 


\section{Abstract}

Background: Recent studies reported that a hot genetic variant, rs641738 within the membrane bound O-acyltransferase domain containing 7(MBOAT7) and transmembrane channel-like 4 (TMC4), was associated with several liver diseases. However, results are still conflicting. We conducted this study to explore the role of MBOAT7-TMC4 rs641738 in the risk of hepatocellular carcinoma (HCC) and persistent hepatitis B virus (HBV) infection.

Methods: We first performed a case-control study by including 779 HCC cases and 1412 cancer-free controls. Controls are consisted of 678 HBV persistent carriers and 734 spontaneously recovered subjects. Rs641738 was genotyped by MassARRAY platform. Results were analyzed by multivariate logistic regression analysis under five genetic models. Second, we conducted a systematic review and metaanalysis to further explore the role of this variant in HCC risk.

Results: Results suggested no association between MBOAT7-TMC4 rs641738 and HCC risk in most genetic models (All P > 0.05), although a marginally significant association was observed in TT vs. $C C(P=0.037)$ and recessive model $(P=0.044)$. Further meta-analysis including $2135 \mathrm{HCC}$ cases and 4388 controls supported that this variant was not related to HCC risk, even in the TT vs. CC and recessive models. Besides, we identified that this variant also had no influence on persistent HBV infection. Conclusion: Our work highlights that MBOAT7TMC4 rs641738 is not associated with the risk of HCC or persistent HBV infection. This study provides some clues to identify the "truth" of potential disease-related genetic factors in the post-genome era.

\section{Background}

Hepatocellular carcinoma (HCC) is a common cancer with rapid progress and high mortality in the world, especially in China [1]. As the fourth common cancer in China, it was estimated to have 466100 new cases in 2015[2]. Environmental factors such as infection of hepatitis $B$ virus (HBV) are confirmed to be a crucial pathogenesis of $\mathrm{HCC}[3]$. Increasing evidence has revealed that genetic factors also play an important role in the development of HCC [4].

HCC is a complicated disease with high genetic heterogeneity. Candidate gene researches and genome-wide association studies (GWASs) have found hundreds of genes and loci associated with HCC risk. How to identify the "truth" of these genetic factors is becoming an urgent issue in the post-genome era. In 2015, a GWAS study reported a genetic variant near the membrane bound O-acyltransferase domain containing 7(MBOAT7) gene, rs641738, C>T, increasing the risk of alcohol-related cirrhosis [5]. Later it is also reported to be located in the transmembrane channel-like 4 (TMC4) gene. In 2016, Thabet demonstrated that MBOAT7-TMC4 rs641738 could be a risk factor of hepatic inflammation and liver fibrosis[6]. Subsequent studies further investigated the association between this variant and HCC risk, but led to a conflicting conclusion [7-9].

Here, we first conducted a case-control study including $779 \mathrm{HCC}$ cases and 1412 cancer-free controls, aiming to explore the effects of MBOAT7-TMC4 rs641738 on HCC risk in a Chinese population. Then we performed a systematic review and meta-analysis to further explore the role of this variant and validate our results.

\section{Methods}

\section{Study Subjects}

This case-control study included 779 HCC cases and 1412 cancer-free controls. Cases were pathologically confirmed and enrolled between January 2014 and June 2016 at Tongji Hopital of Huazhong University of Science and Technology (HUST), central China. Controls are consisted of 678 hepatitis B virus (HBV) persistent carriers and 734 spontaneously recovered subjects, who were recruited from a health screening in the same hospital during the same period as the cases were included. HBV persistent carriers were people who were positive for both of HBsAg and HBcAb, but negative for anti-HCV. Spontaneously recovered subjects were people who were negative for both of HBsAg and anti-HCV, but positive for both of HBcAb and HBsAb. All subjects were unrelated Han Chinese from Wuhan and the surrounding regions. Cases and controls were frequency-matched for sex and age. At recruitment, a 2-ml peripheral blood sample and a written informed consent were collected from each subject, and demographic information (i.e., sex, age, smoking and drinking status) were also obtained by questionnaire. Definitions of smoking and drinking status have been detailed previously $[10,11]$. This study was approved by the institutional ethics committee of Tongji Hospital, Tongji Medical College of HUST.

\section{Serological Testing}

Page 2/10 
Enzyme-linked-immunosorbent assay (ELISA) was applied to detect serum HBsAg, HBsAb, HBcAb and anti-HCV (IMX; Abbott Diagnostics, USA). There were three positive controls, two negative controls and one blank control in each reaction plate. About $5 \%$ of the samples were randomly chosen for repetition, and results were $100 \%$ concordant.

\section{Genotyping}

Genomic DNA was extracted from leucocyte pellets of 2-ml peripheral blood by the coagulated blood DNA mini-extraction kit (DP6101, BioTeke Corporation, China). MBOAT7-TMC4 rs641738 was genotyped by the Sequenom MassARRAY iPLEX Platform (SEQUENOM, CA, USA). All assays were conducted using 384-well plate with positive and negative controls for each plate, without the information of the disease status of samples. We randomly selected $5 \%$ of the samples as duplicate sets, and got a $100 \%$ concordance rate. The average call rate of this variant was $99.7 \%$.

\section{Statistical Analysis}

A goodness-of-fit $\chi^{2}$ test was applied to assess the Hardy-Weinberg equilibrium (HWE) in controls. Differences between cases and controls are examined by independent $t$ test, ANONVA analysis or Pearson's $\chi^{2}$ test according to the category of variables. The risk of HCC or HBV persistent infection was estimated by odds ratio (OR) and $95 \%$ confidence interval (Cl), using a multivariate logistic regression analysis after adjusting for age, sex, smoking and drinking status. A two-tailed $P$ value $<0.05$ was considered statistically significant. All the analyses above were performed by IBM SPSS Statistics version 20.0 (Chicago, IL, USA). In addition, we calculated the statistical power of this study by Power V3.0[12]. Based on our sample size to detect an OR of 1.30, a power of 0.95 was estimated for MBOAT7-TMC4 rs641738.

\section{Meta-analysis}

This meta-analysis was performed according to the guidelines of the Preferred Reporting Items for Systematic Review and Meta-analyses (PRISMA)[13].

\section{Literature search, Study Selection and Data Extraction}

A comprehensive literature search was conducted through PubMed, Embase and Web of Science up to August, 2019 without any restrictions. The search items included "MBOAT7", "TMC4", "rs641738", and "hepatocellular carcinoma". References from identified publications were also reviewed for obtaining potential relevant studies.

The inclusion criteria was as follows: (1) a case-control or cohort study to evaluate the association between MBOAT7-TMC4 rs641738 and HCC risk, and (2) providing ORs and $95 \% \mathrm{Cls}$, or allele frequency and/or genotypes of this variant. A study was excluded if it met one of the following criteria: (1) review, meta-analysis, comment or conference abstract; (2) insufficient data to estimate OR and 95\%Cl; (3) a deviation from HWE in controls; and (4) studies with overlapped data. If a study contained overlapped data with another, we kept the one with larger sample size.

Data was extracted from each included study as follows: first author, publication year, country, ethnicity, gender, age, sample size, genotype distribution, genotyping method, adjustment, and HWE information. Two authors independently conducted the work above. Disagreement was resolved by discussion. The quality of each included study was evaluated by the Newcastle-Ottawa scale (NOS)[14]. Quality evaluation was not an exclusion criterion for eligible studies (Additional file 1).

\section{Statistical Analysis for Meta-analysis}

We used ORs and $95 \% \mathrm{Cls}$ to estimate the association between MBOAT7-TMC4 rs641738 and HCC risk. Multivariate-adjusted OR and $95 \% \mathrm{Cl}$ was preferentially extracted if available, otherwise unadjusted $\mathrm{OR}$ and $95 \% \mathrm{Cl}$ was calculated instead. Between-study heterogeneity was examined by $Q$ test and $P^{2}$ statistic. If $P<0.10$ or $P^{2}>50 \%$, it was considered a significant heterogeneity and applied the random-effects model; otherwise the fixed-effects model was used. To evaluate the quality, we also performed the sensitivity analysis and publication test $[15,16]$. The meta-analysis was conducted via Stata 12.0 software (College Station, TX, USA). 


\section{Results}

\section{Subject Characteristics}

A total of 779 HCC cases, 678 HBV persistent carriers and 734 spontaneously recovered subjects were included in this study. Demographic characteristics are summarized in Table 1. In these three groups, the male proportion of the three groups was $70.6 \%, 68.7 \%$ and $68.1 \%$, respectively; and the mean age was $53.20 \pm 12.49,52.27 \pm 11.45$ and $52.28 \pm 12.89$ years, respectively. There was no significant difference among the three groups on sex $(P=0.551)$, age $(P=0.240)$, smoking $(P=0.071)$ and drinking status $(P=0.091)$. Genotypes of $M B O A T 7$ TMC4 rs641738 in all groups did not deviate from HWE (HCC: $P=0.485$; HBV persistent carriers: $P=0.133$; spontaneously recovered subjects: $P=0.769)$.

\section{Association of MBOAT7-TMC4 rs641738 with Risk of HCC and Persistent HBV Infection}

We performed four comparisons under five genetic models and showed the results in Table 2. In the comparison of HCC cases vs. all controls, we found that after adjusting for sex, age, smoking and drinking status, MBOAT7-TMC4 rs641738 did not confer any increased risk of HCC risk in dominant, additive or allelic model (All $P>0.05$ ). Although this variant seemed a risk factor of HCC in two models, the results were marginally significant (TT vs. CC: $P=0.037$; Recessive: $P=0.044$ ). Similar results were also observed in the further comparison of HCC vs. HBV persistent carriers. When we set spontaneously recovered subjects as controls, we observed that MBOAT7-TMC4 rs641738 was not associated with HCC risk (All $P>0.10$ ). To explore whether this variant influences the risk of persistent HBV infection, we conducted a further comparison between HBV persistent carriers and spontaneously recovered subjects. Results suggested that this variant was also unrelated to persistent infection or clearance of $\mathrm{HBV}($ All $P>0.30)$.

\section{Meta-analysis of the Association between MBOAT7-TMC4 rs641738 and HCC Risk}

In addition, we conducted a systematic review and meta-analysis to further examine our results. Initially, we got 87 records by searching PubMed, Embase and Web of Science, and excluded 79 records by title and abstract review. After assessing the full-text articles, we further excluded five studies due to deviation from HWE $(n=1)[9]$, insufficient data to estimate ORs $(n=1)$ [17], or having overlapped data $(n=3)$ [18-20]. Finally, we included three eligible articles [6-8] and this study for meta-analysis (Additional file 2).

A total of $2135 \mathrm{HCC}$ cases and 4388 controls were included. Characteristics are detailed in Table 3. We first applied a meta-analysis under the allelic model (Figure 1). Pooled results demonstrated that MBOAT7-TMC4 rs641738 was not associated with $\mathrm{HCC}$ risk (OR $=1.10,95 \% \mathrm{Cl}$

$=0.99-1.23, P_{\text {hetergeneity }}=0.453, P=0 \%$ ), which was stable suggested by the sensitivity analysis (Additional file 3 ). Egger's or Begg's test did not suggest any publication bias. Considering that marginally significant results were observed in our study, we further applied metaanalyses in the TT vs. CC and recessive models. As shown in Table 4, pooled results in these models also consistently and stably showed that there was no association between MBOAT7-TMC4 rs641738 and HCC risk.

\section{Discussion}

In this study, we conducted a case-control study including 799 HCC cases and 1412 controls to investigate the effect of MBOAT7-TMC4 rs641738 on HCC risk. Although this variant showed a marginally significant association in TT vs. CC $(P=0.037)$ and recessive model $(P=$ 0.044), the other genetic models all demonstrated non-significant results. We further performed a systematic review and meta-analysis to explore the role of MBOAT7-TMC4 rs641738 in the susceptibility to HCC. A total of $2135 \mathrm{HCC}$ cases and 4388 controls were included. Results consistently suggested that MBOAT7-TMC4 rs641738 was not associated with HCC risk, even in the TT vs. CC and recessive models. In addition, our study revealed that this variant was also not related to persistent HBV infection in all genetic models. To the best of our knowledge, it is the first study to investigate the association between MBOAT7-TMC4 rs641738 and the risk of HCC and persistent HBV infection in Asians.

The rs641738 variant is mapped to the 500-bp downstream of MBOAT7 and likewise located at exon 1 of the TMC4 gene. Therefore, previous studies refer to it as MBOAT7 or MBOAT7-TMC4 variant. This variant was first identified as a risk locus for alcohol-related cirrhosis [5], then reported to be associated with nonalcoholic fatty liver disease (NAFLD)/nonalcoholic steatohepatitis (NASH) [21] and liver fibrosis 
[6]. In 2016, Thabet first investigated the association of MBOAT7-TMC4 rs641738 with HCC risk, but did not fond a significant result[6]. Subsequently, Donati contradicted the conclusion and showed that this variant predisposed to HCC in NAFLD subjects [9]. However, the significant association was not confirmed by later studies [6-8]. Even in the NAFLD cohort, MBOAT7-TMC4 rs641738 did not show any association with the risk of Matteoni type 4 or NASH-HCC [17]. Additionally, a recent meta-analysis revealed that this variant was not related to the susceptibility of NAFLD [22]. In view of a small sample size of the previous studies, we performed this case-control and meta-analysis, and finally confirmed that MBOAT7-TMC4 rs641738 was not associated with HCC risk.

Persistent HBV infection is a crucial risk factors contributing to HCC in China. However, studies about the effect of MBOAT7-TMC4 rs641738 on HBV infection is limited up to date. Here, we also explored the association between MBOAT7-TMC4 rs641738 and persistent HBV infection. Results exhibited that this variant was not related to spontaneous clearance of HBV, which was in concordance with a previous report from a Moroccan cohort[23]. Interestingly, Thabet reported that MBOAT7-TMC4 rs641738 influenced hepatic inflammation and fibrosis in patients with persistent HBV infection [24]. A possible explanation is that this variant's effect on liver disease is not associated with HBV, or it is not the causal variant.

Nowadays, the role of MBOAT7-TMC4 rs641738 in liver diseases is still conflicting. MBOAT7 encodes a lysophosphatidylinositol acyltransferase involved in phospholipid metabolism. TMC4 encodes a member protein of the calcium-dependent chloride channels, which are widely expressed in epithelia of many tissues, and exist in both plasma membrane and intracellular compartments [25]. Some studies showed that rs641738 reduced mRNA and hepatic MBOAT7 expression, but some studies refuted it $[9,24,26]$. Similar contradictions are also reported in fibrosis and NAFLD $[23,24,26]$. In fact, the exact biological mechanisms of MBOAT7 and TMC4 on liver diseases remain elusive. So whether and how MBOAT7-TMC4 rs641738 influences liver diseases is doubtful. Nonetheless, our study and a recent meta-analysis support that this variant is not associated with the risk of HCC and NAFLD, respectively.

There are some limitations in our study. First, this is a hospital-based case-control study, which might be influenced by selection bias. However, our multivariate analysis, power calculation and subsequent meta-analysis ensured accuracy of our conclusion. Second, our metaanalysis only included four studies due to limited number of relevant studies, which were not performed further stratified analyses according to factors including age, sex, smoking or other lifestyle habits. Even so, the results of meta-analysis were reliable because of robustness, no publication bias and low heterogeneity.

\section{Conclusion}

In summary, our case-control study and meta-analysis highlight that MBOAT7-TMC4 rs641738 is not associated with HCC risk. Besides, our work also suggests no relationship of this variant with persistent HBV infection in a Chinese population. Further studies are required to validate our results.

\section{Abbreviations}

MBOAT7, membrane bound 0-acyltransferase domain containing 7; TMC4, transmembrane channel-like 4; HCC, hepatocellular carcinoma; HBV, hepatitis B virus; GWAS, genome-wide association study; HUST, Huazhong University of Science and Technology; HWE, Hardy-Weinberg equilibrium; OR, odds ratio; Cl, confidence interval; PRISMA, Preferred Reporting Items for Systematic Review and Meta-analyses; NOS, Newcastle-Ottawa scale; SD, standard deviation; NAFLD, nonalcoholic fatty liver disease; NASH, nonalcoholic steatohepatitis.

\section{Declarations}

\section{Ethics approval and consent to participate}

This study was approved by the institutional ethics committee of Tongji Hospital, Tongji Medical College of HUST (Wuhan, China). All procedures were in accordance with the approved guidelines and principles of Helsinki Declaration and its later amendments or comparable ethical standards of 1975 . All participants provided written informed consent.

\section{Consent for publication}

Not applicable.

\section{Availability of data and materials}


All data generated or analyzed during this study are available from the corresponding author upon reasonable request. But those clinical data are not available for authors have an ethical and legal responsibility to respect participant's rights to privacy and to protect their identity.

\section{Competing interests}

The authors declare that they have no competing interests.

\section{Funding}

This work was supported by National Natural Science Foundation of China (NSFC-81601839 to NS, and NSFC-81602407 to LL).

\section{Authors' contributions}

PW finished the main part experiment and data analysis, and drafted this manuscript; YZ and YG finished the sample collection. YZ, LL and YG finished the literature search and selection. LL and RZ did part of the data collection and analysis. NS designed the project and reviewed the manuscript. LL and NS acquired the funding. All authors have read and approved this manuscript.

\section{Acknowledgments}

We are grateful to subjects who participated in this study.

\section{References}

1.Siegel RL, Miller KD, Jemal A: Cancer statistics, 2019. CA: a cancer journal for clinicians 2019, 69(1):7-34.

2.Chen W, Zheng R, Baade PD, Zhang S, Zeng H, Bray F, Jemal A, Yu XQ, He J: Cancer statistics in China, 2015. CA: a cancer journal for clinicians 2016, 66(2):115-132.

3.Sagnelli E, Macera M, Russo A, Coppola N, Sagnelli C: Epidemiological and etiological variations in hepatocellular carcinoma. Infection 2019.

4.Khemlina G, Ikeda S, Kurzrock R: The biology of Hepatocellular carcinoma: implications for genomic and immune therapies. Molecular cancer 2017, 16(1):149.

5.Buch S, Stickel F, Trepo E, Way M, Herrmann A, Nischalke HD, Brosch M, Rosendahl J, Berg T, Ridinger M et al: A genome-wide association study confirms PNPLA3 and identifies TM6SF2 and MBOAT7 as risk loci for alcohol-related cirrhosis. Nature genetics 2015, 47(12):14431448 .

6.Thabet K, Asimakopoulos A, Shojaei M, Romero-Gomez M, Mangia A, Irving WL, Berg T, Dore GJ, Gronbaek H, Sheridan D et al: MBOAT7 rs641738 increases risk of liver inflammation and transition to fibrosis in chronic hepatitis C. Nature communications 2016, 7:12757.

7.Stickel F, Buch S, Nischalke HD, Weiss KH, Gotthardt D, Fischer J, Rosendahl J, Marot A, Elamly M, Casper M et al: Genetic variants in PNPLA3 and TM6SF2 predispose to the development of hepatocellular carcinoma in individuals with alcohol-related cirrhosis. The American journal of gastroenterology 2018, 113(10):1475-1483.

8.Raksayot M, Chuaypen N, Khlaiphuengsin A, Pinjaroen N, Treeprasertsuk S, Poovorawan Y, Tanaka Y, Tangkijvanich P: Independent and additive effects of PNPLA3 and TM6SF2 polymorphisms on the development of non-B, non-C hepatocellular carcinoma. Journal of gastroenterology 2019, 54(5):427-436.

9.Donati B, Dongiovanni P, Romeo S, Meroni M, McCain M, Miele L, Petta S, Maier S, Rosso C, De Luca L et al: MBOAT7 rs641738 variant and hepatocellular carcinoma in non-cirrhotic individuals. Scientific reports 2017, 7(1):4492.

10.Chen X, Wang Y, Chen X, Cheng K, Li J, Lou J, Ke J, Yang Y, Gong Y, Zhu Y et al: Genetic variants in the regulatory region of SLC10A1 are not associated with the risk of hepatitis $B$ virus infection and clearance. Infection, genetics and evolution: journal of molecular epidemiology and evolutionary genetics in infectious diseases 2016, 44:495-500. 
11.Shen N, Li L, Xu W, Tian J, Yang Y, Zhu Y, Gong Y, Ke J, Gong J, Chang J et al: A missense variant in PTPN12 associated with the risk of colorectal cancer by modifying Ras/MEK/ERK signaling. Cancer epidemiology 2019, 59:109-114.

12.Lubin JH, Gail MH: On power and sample size for studying features of the relative odds of disease. American journal of epidemiology 1990, 131(3):552-566.

13.Moher D, Liberati A, Tetzlaff J, Altman DG: Preferred reporting items for systematic reviews and meta-analyses: the PRISMA statement. PLoS medicine 2009, 6(7):e1000097.

14.Stang A: Critical evaluation of the Newcastle-Ottawa scale for the assessment of the quality of nonrandomized studies in meta-analyses. European journal of epidemiology 2010, 25(9):603-605.

15.Begg CB, Mazumdar M: Operating characteristics of a rank correlation test for publication bias. Biometrics 1994, 50(4):1088-1101.

16.Egger M, Davey Smith G, Schneider M, Minder C: Bias in meta-analysis detected by a simple, graphical test. BMJ 1997, 315(7109):629634.

17.Kawaguchi T, Shima T, Mizuno M, Mitsumoto Y, Umemura A, Kanbara Y, Tanaka S, Sumida Y, Yasui K, Takahashi M et al: Risk estimation model for nonalcoholic fatty liver disease in the Japanese using multiple genetic markers. PloS one 2018, 13(1):e0185490.

18.Donati B, Dongiovanni P, Miele L, Rosso C, Maier S, Petta S, Meroni M, De Luca L, Grimaudo S, Romagnoli R et al: MBOAT7 locus rs641738 variant predisposes to hepatocellular carcinoma in nonalcoholic fatty liver. Digestive and Liver Disease 2016, 48(SUPPL. 1):e7-e8.

19.Stickel F, Buch S, Janet F, Rosendahl J, Morgan MY, Nischalke HD, Lammert F, Casper M, Zopf S, Marhenke S et al: Genetic variation of PNPLA3 and TM6SF2 associate with hepatocellular carcinoma in patients with alcohol-related cirrhosis. Journal of hepatology 2017, 66(1 Supplement 1):S174.

20.Stickel F, Buch S, Rosendahl J, Nischalke H-D, Lammert F, Casper M, Vogel A, Deltenre P, Eyer F, Gotthardt D et al: OWE-016 Genetic variants in PNPLA3 and TM6SF2 predispose to hepatocellular carcinoma in patients with alcohol-related cirrhosis. Gut 2018, 67(Suppl_1):A106.

21.Mancina RM, Dongiovanni P, Petta S, Pingitore P, Meroni M, Rametta R, Boren J, Montalcini T, Pujia A, Wiklund O et al: The MBOAT7-TMC4 Variant rs641738 Increases Risk of Nonalcoholic Fatty Liver Disease in Individuals of European Descent. Gastroenterology 2016, 150(5):1219-1230 e1216.

22.Xia Y, Huang CX, Li GY, Chen KH, Han L, Tang L, Luo HQ, Bao MH: Meta-analysis of the association between MBOAT7 rs641738, TM6SF2 rs58542926 and nonalcoholic fatty liver disease susceptibility. Clinics and research in hepatology and gastroenterology 2019.

23.Ezzikouri S, Elfihry R, Chihab H, Elmessaoudi-Idrissi M, Zaidane I, Jadid FZ, Karami A, Tahiri M, Elhabazi A, Kabine M et al: Effect of MBOAT7 variant on hepatitis B and C infections in Moroccan patients. Scientific reports 2018, 8(1):12247.

24. Thabet K, Chan HLY, Petta S, Mangia A, Berg T, Boonstra A, Brouwer WP, Abate ML, Wong VW-S, Nazmy M et al: The membrane-bound Oacyltransferase domain-containing 7 variant rs641738 increases inflammation and fibrosis in chronic hepatitis B. Hepatology (Baltimore, Md) 2017, 65(6):1840-1850.

25.Li X, Weinman SA: Chloride Channels and Hepatocellular Function: Prospects for Molecular Identification. Annual Review of Physiology 2002, 64(1):609-633.

26.Sookoian S, Flichman D, Garaycoechea ME, Gazzi C, Martino JS, Castano GO, Pirola CJ: Lack of evidence supporting a role of TMC4rs641738 missense variant-MBOAT7-intergenic downstream variant-in the Susceptibility to Nonalcoholic Fatty Liver Disease. Scientific reports $2018,8(1): 5097$.

\section{Tables}

Table 1. The characteristics of the included subjects 


\begin{tabular}{|c|c|c|c|c|c|}
\hline iables & HCC, N (\%) & $\begin{array}{l}\text { HBV persistent carriers, } \mathrm{N} \\
(\%)\end{array}$ & $\begin{array}{l}\text { Spontaneously recovered subjects, N } \\
(\%)\end{array}$ & $\chi^{2} / F$ & $P$ \\
\hline$\overline{\mathrm{al}}$ & 779 & 678 & 734 & & \\
\hline$\tau$ & & & & 1.191 & 0.551 \\
\hline Male & $550(70.6)$ & 466 (68.7) & $500(68.1)$ & & \\
\hline Female & 229 (29.4) & $212(31.3)$ & $234(31.9)$ & & \\
\hline э (mean \pm & $\begin{array}{l}53.20 \pm \\
12.49\end{array}$ & $5227+1145$ & $5228+1289$ & 1428 & 0.240 \\
\hline oking status & & & & 5.280 & 0.071 \\
\hline Smokers & $301(38.6)$ & $243(35.8)$ & $242(33.0)$ & & \\
\hline Non-smokers & $478(61.4)$ & $435(64.2)$ & $492(67.0)$ & & \\
\hline nking status & & & & 4.803 & 0.091 \\
\hline Drinkers & $270(34.7)$ & $265(39.1)$ & $291(39.6)$ & & \\
\hline Non-drinkers & $509(65.3)$ & $413(60.9)$ & $443(60.4)$ & & \\
\hline
\end{tabular}

Table 2. The effects of MBOAT7-TMC4 rs641738 on persistent HBV infection and HCC

\begin{tabular}{|c|c|c|c|c|c|c|c|}
\hline$\overline{\mathrm{ees}}$ & $\begin{array}{l}\mathrm{HCC}, \\
\mathrm{N}(\%)\end{array}$ & $\begin{array}{l}\text { HBV persistent } \\
\text { carriers, } \mathrm{N}(\%)\end{array}$ & $\begin{array}{l}\text { Spontaneously } \\
\text { recovered subjects, N } \\
\text { (\%) }\end{array}$ & $\begin{array}{l}\text { Adjusted OR } \\
(95 \% \mathrm{CI}), P^{a}\end{array}$ & $\begin{array}{l}\text { Adjusted OR } \\
(95 \% \mathrm{CI}), P^{b}\end{array}$ & $\begin{array}{l}\text { Adjusted OR } \\
(95 \% \mathrm{CI}), P^{C}\end{array}$ & $\begin{array}{l}\text { Adjusted OR } \\
(95 \% \mathrm{CI}), P^{d}\end{array}$ \\
\hline & 426 & & & & & & \\
\hline & (54.7) & $380(56.1)$ & $420(57.7)$ & Reference & Reference & Reference & Reference \\
\hline & $\begin{array}{l}295 \\
(37.9)\end{array}$ & $264(39.0)$ & $264(36.3)$ & $\begin{array}{l}1.05(0.87- \\
1.27), 0.604\end{array}$ & $\begin{array}{l}1.00(0.82- \\
1.24), 0.981\end{array}$ & $\begin{array}{l}1.10(0.89- \\
1.37), 0.370\end{array}$ & $\begin{array}{l}1.11(0.89- \\
1.38), 0.366\end{array}$ \\
\hline & $\begin{array}{c}58 \\
(7.4)\end{array}$ & 33 (4.9) & $44(6.0)$ & $\begin{array}{l}1.47(1.02- \\
2.12), 0.037\end{array}$ & $\begin{array}{l}1.64(1.04- \\
2.57), 0.033\end{array}$ & $\begin{array}{l}1.35(0.89- \\
2.05), 0.164\end{array}$ & $\begin{array}{l}0.85(0.53- \\
1.36), 0.491\end{array}$ \\
\hline nt & & & & $\begin{array}{l}1.10(0.92- \\
1.32), 0.280\end{array}$ & $\begin{array}{l}1.07(0.87- \\
1.32), 0.541\end{array}$ & $\begin{array}{l}1.14(0.93- \\
1.40), 0.219\end{array}$ & $\begin{array}{l}1.07(0.87- \\
1.32), 0.529\end{array}$ \\
\hline re & & & & $\begin{array}{l}1.44(1.01- \\
2.06), 0.044\end{array}$ & $\begin{array}{l}1.64(1.05- \\
2.55), 0.029\end{array}$ & $\begin{array}{l}1.29(0.86- \\
1.95), 0.218\end{array}$ & $\begin{array}{l}0.81(0.51- \\
1.29), 0.383\end{array}$ \\
\hline : & & & & $\begin{array}{l}1.13(0.98- \\
1.31), 0.094\end{array}$ & $\begin{array}{l}1.13(0.95- \\
1.33), 0.172\end{array}$ & $\begin{array}{l}1.13(0.96- \\
1.34), 0.138\end{array}$ & $\begin{array}{l}1.02(0.85- \\
1.21), 0.851\end{array}$ \\
\hline od & l $(\mathrm{T}$ & & & $\begin{array}{l}1.13(0.98- \\
1.30), 0.095\end{array}$ & $\begin{array}{l}1.12(0.95- \\
1.33), 0.175\end{array}$ & $\begin{array}{l}1.14(0.96- \\
1.34), 0.135\end{array}$ & $\begin{array}{l}1.02(0.86- \\
1.21), 0.853\end{array}$ \\
\hline
\end{tabular}

${ }^{\text {a }}$ HCC vs. (HBV persistent carriers + spontaneously recovered subjects), adjusted for sex, age, smoking and drinking status.

b HCC vs. HBV persistent carriers, adjusted for sex, age, smoking and drinking status.

${ }^{\mathrm{c}}$ HCC vs. spontaneously recovered subjects, adjusted for sex, age, smoking and drinking status.

${ }^{\mathrm{d}}$ HBV persistent carriers vs. spontaneously recovered subjects, adjusted for sex, age, smoking and drinking status.

Table 3. The characteristics of the included studies 


\begin{tabular}{|c|c|c|c|c|c|c|c|c|c|c|c|}
\hline & Country & Ethnicity & Male, $\mathbf{N}$ & Age (mean & Cases/ & Genotype & $\mathrm{CC} / \mathrm{TC} / \mathrm{TT})$ & Genotyping & Adjustment & HWE & NOS \\
\hline & & & & $\begin{array}{l}\text { mean } \\
\text { [25th, } 75 \text { th } \\
\text { percentile]) }\end{array}$ & & Case & Control & & & & score \\
\hline $\begin{array}{l}\mathrm{t} \\
\text { ) }\end{array}$ & $\begin{array}{l}\text { Multi- } \\
\text { country }\end{array}$ & Caucasian & $\begin{array}{l}\text { Case: } \\
\text { NA; } \\
\text { Control: } \\
1101 \\
(64.5)\end{array}$ & $\begin{array}{l}\text { Case: NA; } \\
\text { Control: } \\
44.9 \text { (38- } \\
52)\end{array}$ & $75 / 1706$ & $24 / 35 / 16$ & $16 / 531 / 822$ & TaqMan & $\begin{array}{l}\text { Age, } \\
\text { gender, } \\
\text { BMI and } \\
\text { Child-Pugh } \\
\text { score }\end{array}$ & 0.288 & 9 \\
\hline ) & Switzerland & Mixed & $\begin{array}{l}\text { Case: } \\
679 \\
\text { (90); } \\
\text { Control: } \\
817 \\
(70)\end{array}$ & $\begin{array}{l}\text { Case: } 61 \pm \\
10 ; \\
\text { Control: } 55 \\
\pm 10\end{array}$ & $751 / 1165$ & $203 / 363 / 185$ & $185 / 314 / 583$ & TaqMan & $\begin{array}{l}\text { Age, } \\
\text { gender, } \\
\text { BMI, and } \\
\text { type II } \\
\text { diabetes } \\
\text { mellitus }\end{array}$ & 0.934 & 7 \\
\hline yot & Thailand & NA & $\begin{array}{l}\text { Case: } \\
424 \\
(0.8) ; \\
\text { Control: } \\
77 \\
(73.3)\end{array}$ & $\begin{array}{l}\text { HBV-HCC: } \\
62.1 \pm 7.8, \\
\text { HCV-HCC: } \\
62.2 \pm 7.5, \\
\text { NBNC- } \\
\text { HCC: } 63.3 \\
\pm 9.3 ; \\
\text { Control: } \\
50.7 \pm 4.6\end{array}$ & $530 / 105$ & $279 / 213 / 38$ & $38 / 66 / 34$ & TaqMan & No & 0.818 & 6 \\
\hline & China & Chinese & $\begin{array}{l}\text { Case: } \\
550 \\
(70.6) ; \\
\text { Control: } \\
966 \\
(68.4)\end{array}$ & $\begin{array}{l}\text { Case: } 53.2 \\
\pm 12.5 ; \\
\text { Control: } \\
52.3 \pm 12.2\end{array}$ & $779 / 1412$ & $426 / 295 / 58$ & $58 / 800 / 528$ & Massary & $\begin{array}{l}\text { Age, sex, } \\
\text { smoking } \\
\text { and } \\
\text { drinking } \\
\text { status }\end{array}$ & 0.403 & 7 \\
\hline
\end{tabular}

Table 4. Meta-analysis of the association between MBOAT7-TMC4 rs641738 and HCC risk under other genetic models

\begin{tabular}{lllllll}
\hline Genetic model & OR $(95 \% \mathrm{CI})$ & $P_{\text {hetergeneity }}$ & $I^{2}(\%)$ & Sensitivity analysis & $P_{\text {egg }}$ & $P_{\text {begg }}$ \\
\hline Recessive & $1.17(0.98-1.38)$ & 0.623 & 0 & Stable & 0.529 & 0.734 \\
\hline TT vs. CC & $1.18(0.97-1.43)$ & 0.472 & 0 & Stable & 0.548 & 0.734 \\
\hline
\end{tabular}

\section{Figures}




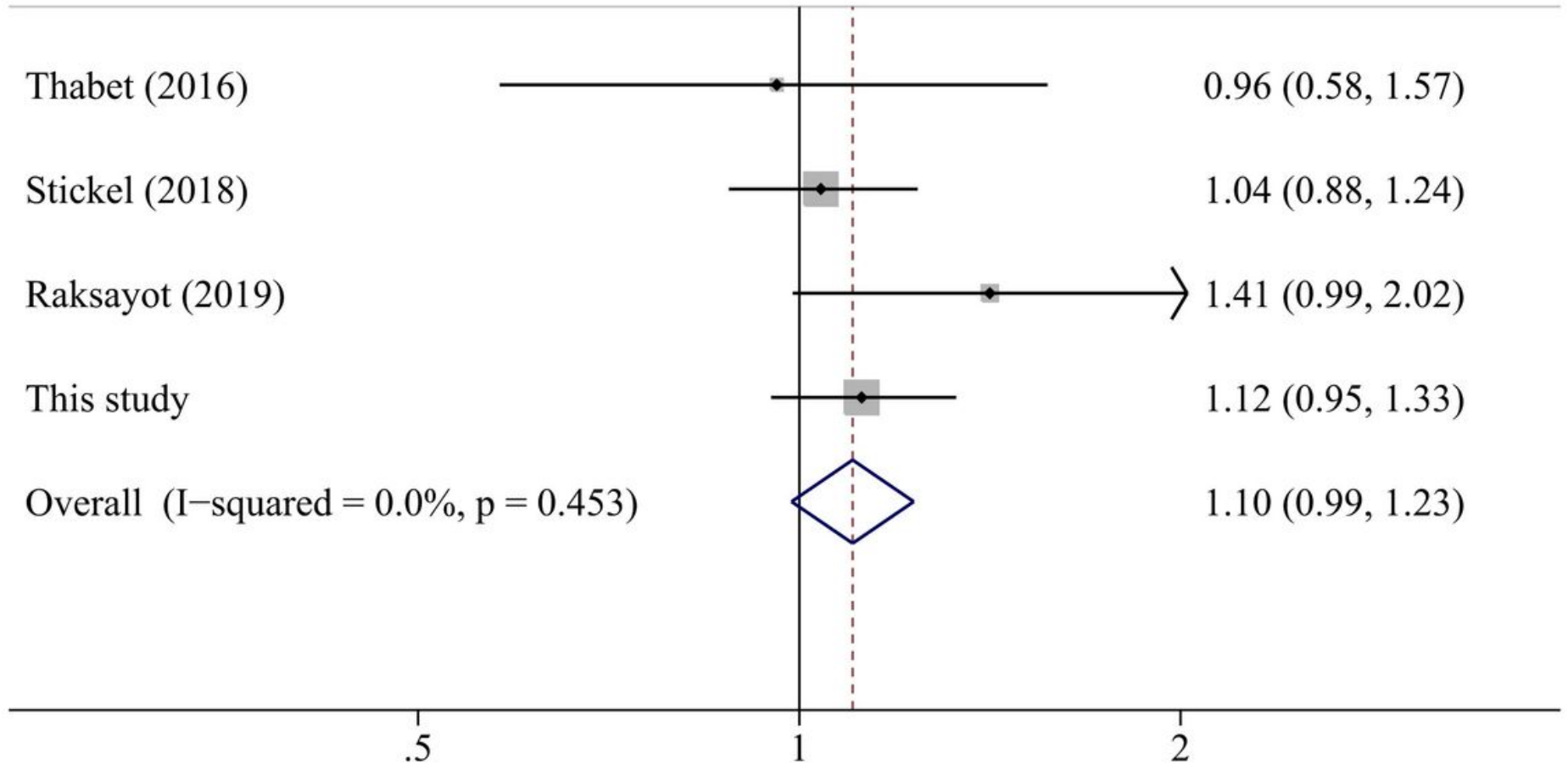

Figure 1

The forest plot of the association between MBOAT7-TMC4 rs641738 and HCC risk under the allelic model.

\section{Supplementary Files}

This is a list of supplementary files associated with this preprint. Click to download.

- Additionalfile3.tif

- Additionalfile1.docx

- Additionalfile2.tif 